\title{
MEMS BASED SINGLE DEVICE “XOR” AND "AND" LOGIC GATES FOR LSI MECHANICAL PROCESSORS OPERATING IN HIGH RADIATION ENVIRONMENTS
}

\author{
Faisal K. Chowdhury ${ }^{1}$, Sijing Han ${ }^{2}$, Daniel Saab ${ }^{2}$ and Massood Tabib-Azar ${ }^{1 *}$ \\ ${ }^{1}$ University of Utah, Salt Lake City, Utah, USA \\ ${ }^{2}$ Case Western Reserve University, Cleveland, Ohio, USA
}

\begin{abstract}
Here we demonstrate the feasibility of MEMS functional devices where a single device functions as a logic gate. This novel approach reduces the number of MEMS devices needed to implement a mechanical processor by $\sim 10 \mathrm{X}$. MEMS processors are suitable for operation in harsh environment in engines and in the presence of ionizing radiations. Generally, MEMS devices have overall lower speed and less reliability than CMOS. By reducing the number of devices needed for a given operation, our approach improves yield, reproducibility, speed and simplifies implementation of MEMS-based circuits such as adders and multiplexers. Specifically, we discuss XOR \& AND gates fabricated on $\mathrm{Si}_{3} \mathrm{~N}_{4}$ \& Polysilicon as bridge materials using W electrodes. The XOR gates with $\sim 1.5 \mathrm{~V}$ turn-on voltage at $50 \mathrm{MHz}$ with $>10^{9}$ cycles of reliable operations and low operational power consumption (leakage current and power $<10^{-9},<1 \mu \mathrm{W}$ ) were tested. We also present data showing the operation of XOR without deterioration at high temperature and in $90 \mathrm{~kW}$ ionizing radiation for 120 minutes. Related circuits such as 2-bit full adder and multiplexer are also discussed.
\end{abstract}

\section{INTRODUCTION}

Simple micro-electro-mechanical on/off switches have been reported in the past for applications in processors and to address power management in scaled VLSI, programming interconnect in FPGA's, biomedical devices where it is desirable to reduce leakage power to prolong implanted battery life, and other applications in harsh environment where CMOS cannot operate due to high temperature or radiation [1-9]. One such case includes operation in the presence of Ionizing Radiation (I-R) in troubled reactors like Chernobyl and Fukushima or at high temperatures encountered inside combustion engines. In these cases silicon channel in CMOS becomes highly conductive due to thermal generation of carriers or due to lattice defect generation caused by I-R radiation over prolonged exposure [1]. Space applications also require radiationhard devices and materials. In some electronic materials such as $\mathrm{SiC}$, the energy required to produce lattice defects is high enabling these materials to withstand I-R longer than $\mathrm{Si}$. In other materials such as InP, defects heal at relatively low temperatures enabling them to recover quickly.

NEMS/MEMS devices are based on mechanical elements that are inherently insensitive to I-R. The I-R causes lattice defects in these devices but these defects do not alter their characteristics the way they affect channel resistance in CMOS. Eventually large defect densities created over extended I-R exposure leads to embrittlement that may affect NEMS/MEMS electrical and switching characteristics. NEMS/MEMS devices also have very low leakage power making them very desirable in biomedical implant devices or other applications requiring very long battery lifetime.

Despite their very high off-to-on resistance (100 G $\Omega$ to 10 $\mathrm{m} \Omega$ ) ratios, and very low off-state leakage currents $\left(<10^{-14} \mathrm{~A}\right)$, MEMS switches tend to be slow $(<1 \mathrm{MHz})$, large $\left(>40 \mu \mathrm{m}^{2}\right)$, and unreliable with limited lifetime of $\sim 10^{6}$ operation cycles [1-4]. In addition, MEMS/NEMS switches have many interesting and challenging issues including: a) contact reliability, b) stiction problem related to release during fabrication and micro-welding during hot-contact operation, c) reliability of flexure structures usually used as part of the switch and d) particulate problems that occur during repeated operations that can lead to switch failure. To address some of these issues, our approach during the last two years have shifted from the CMOS paradigm that uses individual p- and n-MOSFET (PMOS and NMOS) as complementary switches for implementing logic gates to single device functional structures creating a platform for improved density, reliability, speed and fabrication yield. The devices are based on a composite $\mathrm{Si}_{3} \mathrm{~N}_{4} /$ Polysilicon cross-bridge platform with metal contacts designed to provide AND or XOR capability from a single actuating structure. The following sections elaborate on the functional structure design, fabrication process and the results after testing these novel single device XOR and AND gates.

\section{FUNCTIONAL STRUCTURE DESIGN}

The cross-bridge platform shown in figure 1 forms the basis for both XOR and AND gates. It illustrates the electrode design for an XOR gate where metal traces overlap at the intersection area of the bridges and the bridges are actuated by electrostatic attraction between the gate electrodes.

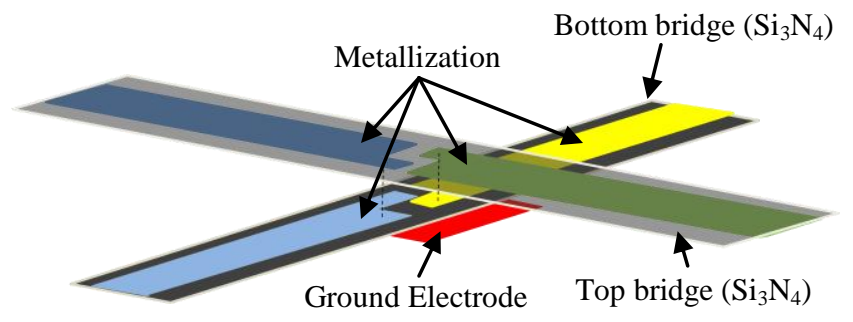

Figure 1: Schematic of MEMS based single device XOR gate. Only the central regions of the bridges are shown. The metallization patterns at the top surface of the bottom bridge and at the bottom surface of the top bridge are matched so that when the two bridges contact each other, the intended function is executed.

Other logic gates can be constructed using similar structures but different metallization/contact patterns as shown in figure 2 for an "AND" gate. In figure 2 the cross sectional view of the XOR and AND device can be seen. The diagram has the two bridges, Gate 1, Gate 2, Drain and Source labeled.

When both gates are low (" 00 ") or when both gates are high ("11") there is no electrostatic attraction (see XOR in figure 2 and figure $3(\mathrm{a})$ ). When either one of the gates are high (" 10 " or " 01 ") electrostatic attraction causes the drain and source to collapse towards each other and thereby make contact. This is the XOR function (ref. to truth table in figure 2). A minor modification of the electrode design produces similar functionality corresponding to AND gate (figure 2 and 3(b)). The AND gate requires that both G1 \& G2 be high at the same time to enable the top bridge to 
experience sufficient electrostatic attraction to cause the bridge to collapse towards the bottom bridge \& thereby make a S-D connection. This is the $\mathrm{ON}$ state. All other conditions translate to OFF state. In the XOR gate, to prevent the drain-source electrodes from causing the attraction between the two bridges their overlapping area is designed to be 4 times smaller than that of the gate electrodes. The same is true for the AND gate with the ratio being slightly lower. The fabrication procedure of these devices is discussed in the following section. Other gates such as NAND and NOT can also be realized in a similar manner. An XOR gate can be converted to NOT gate by fixing one of its inputs at "1". A NAND gate can be produced by NOT(AND) or by using different device structure.

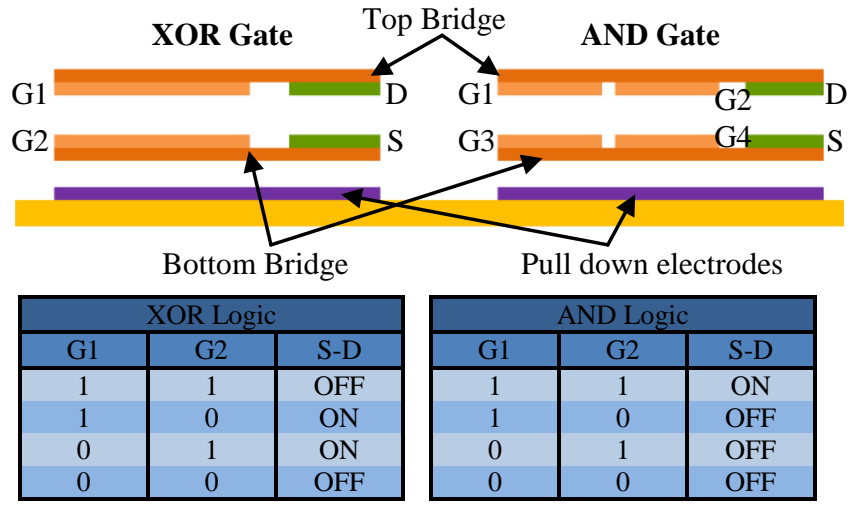

Figure 2: Cross-section of XOR and AND gates. The table shows the truth table corresponding to XOR gate operation

Figure 3 shows optical images of the XOR and AND gates. The common implementation of XOR using 8 individual switches is shown in figure 4 . The factor 8 reduction in device count and associated reduction in number of moving parts and areas lead to 8 times better reliability, at least 4 times faster gate speed and proportionately higher yields. Moreover, multi-input $(>2)$ gates can also be designed using the cross-bridge geometry with two or more metal traces for multiple contact electrodes. A 4-input XOR gate will compress the device count by $\mathrm{x} 24$.
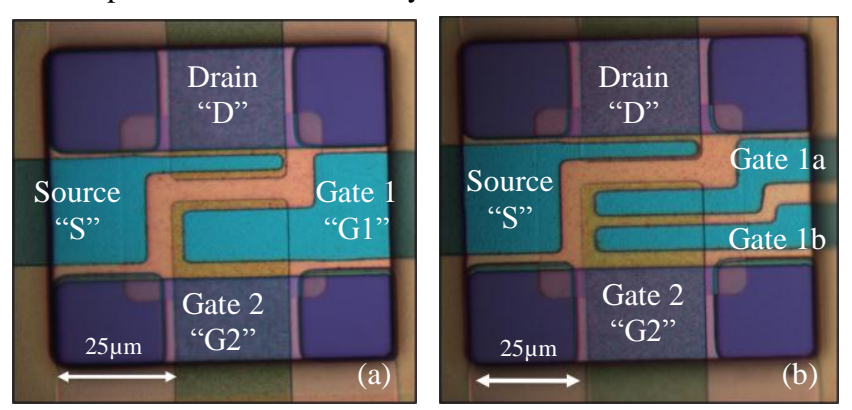

Figure 3: a) Fabricated XOR gate. G1 and G2 are gate electrodes. $S$ and $D$ represent Source/Drain. b) Fabricated AND gate. $S-D$ will connect only if gate $1 a \& 1 b$ are high at the same time.

\section{FABRICATION}

The fabrication process flow is given in figure 5. The conducting silicon wafer was insulated with $100 \mathrm{~nm}$ LPCVD stoichiometric silicon nitride deposited at $780^{\circ} \mathrm{C}$, first tungsten layer (thickness $100 \mathrm{~nm}$ ) sputtered uniformly and patterned to form a "stationary" ground electrode. This electrode was used both as a gate in "MOSFET" like operation of the device as well as to use field effect to separate the two bridges if needed.

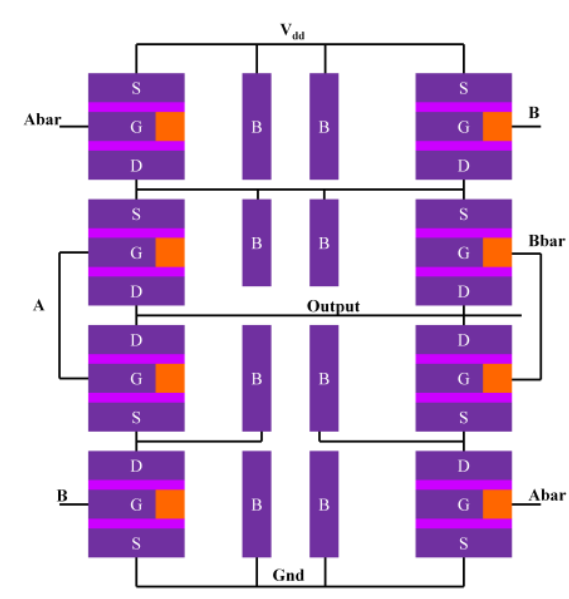

Figure 4: XOR implemented using individual switches require 8 devices each $25 \mu^{2}$ while our single XOR device requires only $25 \mathrm{\mu m}^{2}$ area.

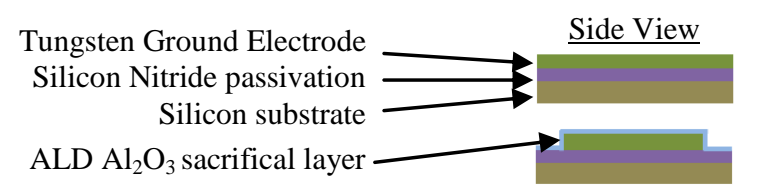

Silicon Nitride Bridge Layer

Tungsten electrode

$\mathrm{ALD} \mathrm{Al}_{2} \mathrm{O}_{3}$ sacrificial layer

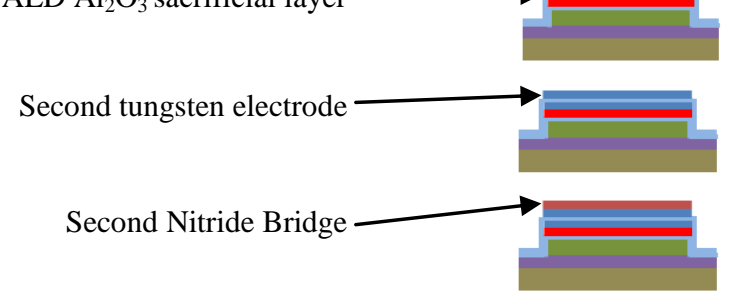

Final device after sacrificial $\mathrm{Al}_{2} \mathrm{O}_{3}$ wet etch

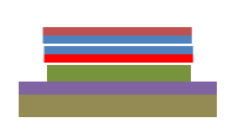

$\underline{\text { Top View }}$

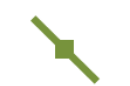

Figure 5: Fabrication process flow for XOR and AND gates. The process flow is the same for other gates.

The $100 \mathrm{~nm}$ tungsten was then capped with 100 layers $(\sim 0.1$ $\mathrm{nm} /$ layer) of thermal $\mathrm{Al}_{2} \mathrm{O}_{3}$ deposited using Fiji Atomic Layer Deposition (ALD) system and a second layer of LPCVD nitride (100nm) was deposited and patterned to form the first nitride bridge. In step 6 , the second layer of tungsten was sputterdeposited and patterned to form the electrodes on the first nitride bridge. ALD $\mathrm{Al}_{2} \mathrm{O}_{3}$ was subsequently used to cap the patterned tungsten and also serve as the sacrificial gap between bottom and top electrode-bridges. This was followed by the third tungsten metallization and patterning to deposit the electrodes that reside under the second nitride bridge. Then, another patterned nitride layer was used to define the top bridge. After fabricating the whole structure that required 8 masks, the ALD layers were sacrificially etched in BOE etchant to "free" the two bridges from each other 
and from the substrate. $\mathrm{H}_{2} \mathrm{O}_{2}$ at room temperature was used as the wet etchant to pattern tungsten resulting in a clean and uniform etch all over the wafer. Silicon Nitride was pattered using a dry etch recipe of $\mathrm{CF}_{4} / \mathrm{O}_{2}$ at $200 \mathrm{~W}$. This also resulted in a clean etch of the nitride bridges. The ALD $\mathrm{Al}_{2} \mathrm{O}_{3}$ layers acted as an effective etch stop for this dry etch recipe.

\section{TESTING AND RESULTS}

The micro-fabricated XOR gate was tested for switching and reliability using an Agilent $4156 \mathrm{C}$ Precision Semiconductor Analyzer, with the current compliance fixed at $1 \mu \mathrm{A}$, coupled to a probe station. A switching voltage of approximately $1.5 \mathrm{~V}$ was observed over repeated cycling as seen in figure 6 .

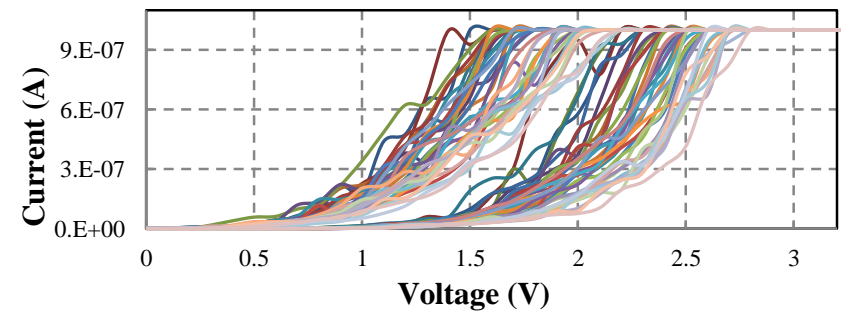

Figure 6: I-V characteristics over a million switching cycles. In this test, the G1 was shorted to D and G2 was shorted to $S$. These contacts are shown in Fig. 2 and the voltage was then applied between the G2-D and G1-S electrodes.

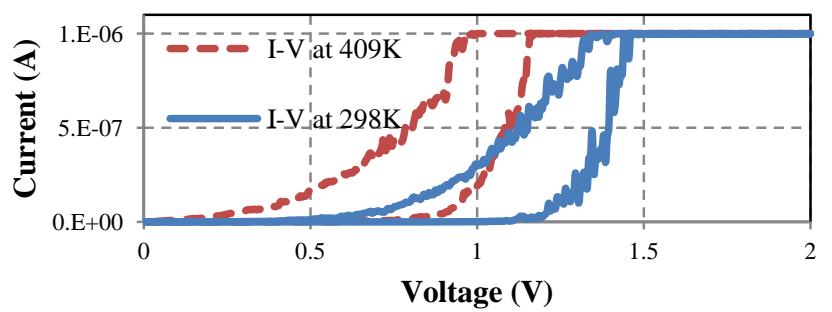

Figure 7: Switching characteristics at elevated temperatures. These tests were performed using the same electrode arrangements used in figure 8.

The devices were also tested under harsh conditions in a chamber with controlled environment where temperature was monitored and maintained as required. The I-V characteristics of the switch at $298 \mathrm{~K}$ and $409 \mathrm{~K}$ are compared in figure 7 . It reveals that the switching characteristics are consistent, even at elevated temperatures, albeit shifted by $\sim 0.5 \mathrm{~V}$.

The setup used to test the device's logic functionality is schematically shown in figure 8 . As seen from the switching characteristics, the two gate regions attract each other only when one of them is high. No actuation was observed when either both gates are low or both gates are high. At the ON state (when the bridges contact each other), the drain and source electrodes contact each other and produce the desired output. This simple structure operates as an XOR gate. The fundamental design of the device with " $D$ " and "S" electrodes situated on separate bridges lead to very small leakage current. The electrodes had dimensions on the order of $\sim 2 \mu \mathrm{m}$ at the points of contact while the leakage current was less than $10^{-9} \mathrm{~A}$. The leakage power is shown in figure 9 .

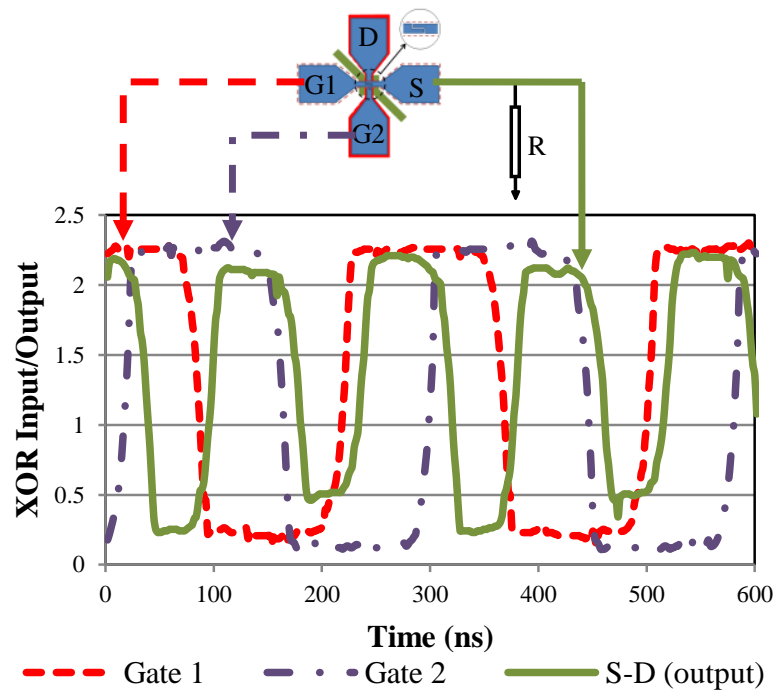

Figure 8: Voltage transitions of a single XOR device. S-D transitions to HIGH only when either G1 or G2 are high. When both $G 1 \& G 2$ are LOW (or HIGH) together, S-D transitions to LOW.

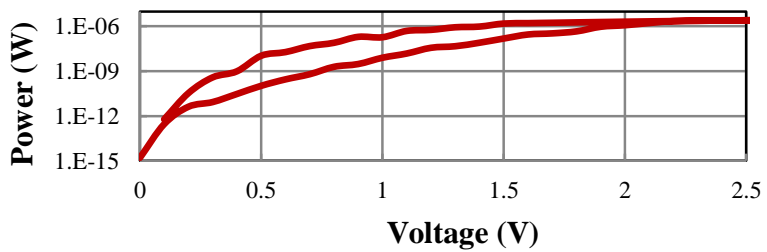

Figure 9: Graph showing leakage power characteristic of the fabricated XOR gate $(\sim 1 \mu W)$.

Figures 10-11 shows the I-V characteristics of a MOSFET and an XOR gate connected as a switch in $90 \mathrm{~kW}$ ionizing radiation environment. After 120 minutes of exposure the MOSFET's I $I_{D S}$ increased by an order of magnitude and its channel became permanently conducting (figure 10 (b)). At this point its gate could no longer control the channel current. In the case of XOR gate, the I-Vs are not affected much by the radiation, as seen in figure 11 . However, the switch characteristics changed but the device continued to operate with clear "on" and "off" states.
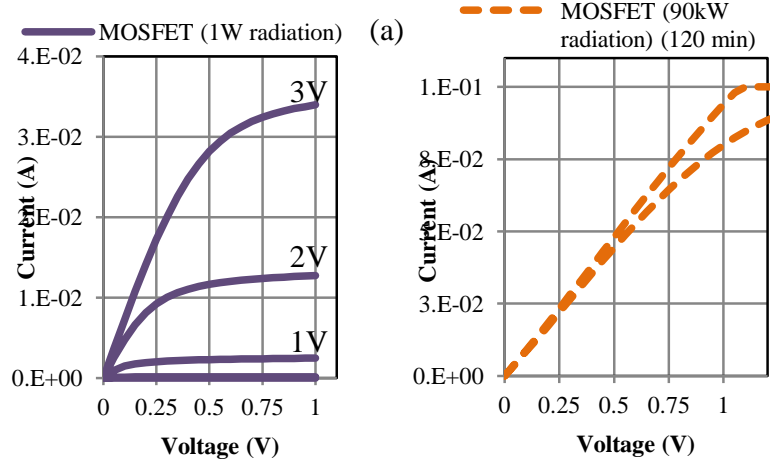

Figure 10: a) MOSFET $I_{d s}-V_{d s}$ at $1 \mathrm{~W}$ ionizing radiation, $1 \mathrm{~min}, b$ ) its $I_{d s}-V_{d s}$ at $90 \mathrm{~kW}$ ionizing radiation, 120min 


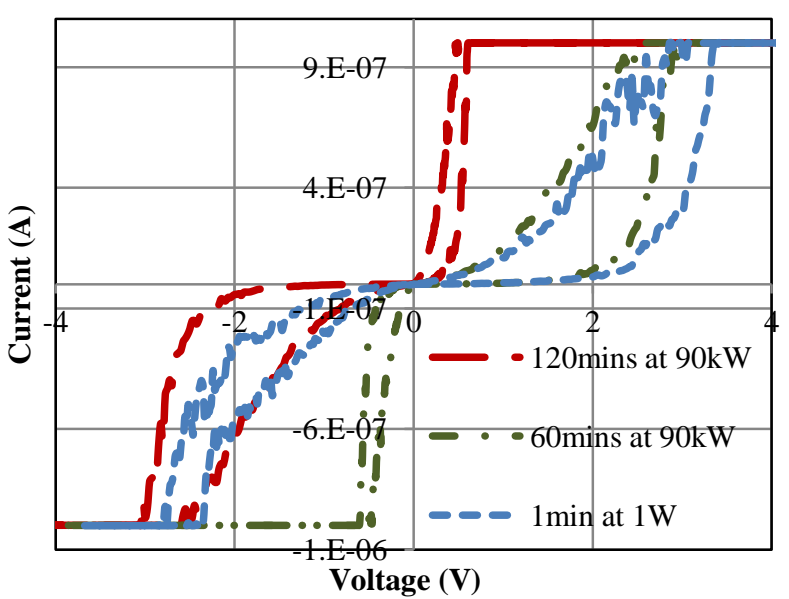

Figure 11: MEMS Switch $I_{d s}-V_{d s}$ at increasing ionizing radiation powers and at prolonged time, (1W/1min to 90kW/120min). Clear "on" \& "off" states are still discernible even after prolonged exposure to I-R.

The TRIGA reactor at University of Utah was used in these experiments. At $90 \mathrm{~kW}$, typical neutron flux is $\sim 3 \times 10^{12}$ neutrons $/ \mathrm{cm}^{2}$-sec. The energy range of the neutrons vary from $0.025 \mathrm{eV}$ to $10 \mathrm{MeV}$, but most of them have neutron energies less than $1 \mathrm{MeV}$. In general, alpha particles do not exist in the reactor core except for inside the fuel element which has an average energy of $6 \mathrm{MeV}$. It is known that Gamma rays exist ubiquitously in the reactor core. Approximate gamma flux is on order of $10^{13}$ gamma $/ \mathrm{cm}^{2}$-sec (or higher). Gamma particles' energy ranges from approximately several $\mathrm{keV}$ to $3 \mathrm{MeV}$. Beta particles' flux at $90 \mathrm{~kW}$ is approximately $10^{13} \mathrm{beta} / \mathrm{cm}^{2}-\mathrm{sec}$ and typical energy of a beta particle is between $100 \mathrm{keV} \sim 1500 \mathrm{keV}$.

Figure 12 (a)-(c) illustrates optical images of a 1-bit multiplexer chip with 4 AND gates, a 1-bit adder chip with 3 XOR and 2 AND gates and a 2-bit full adder circuit utilizing 6 XOR and 4 AND gates that were fabricated and are being tested.

\section{CONCLUSION}

This article presented functional MEMS/NEMS structures to implement logic gates in a single device instead of using individual switches commonly employed in CMOS. Given that 6-14 switches are typically needed in logic gates, the functional structures reduce the device count leading to better reliability, yield, speed and overall better characteristics (sub-threshold characteristics, smaller turn-on/off voltage variations, etc.). The design, fabrication and characterization of XOR gate as a specific example of a functional structure and AND gates was also discussed. Also, operational characterization data showed a $\sim 2 \mathrm{~V}$ actuation, $<1 \mu \mathrm{W}$ leakage power consumption and an operational lifetime of $\sim 10^{9}$ cycles. Moreover, harsh environment operation at elevated temperatures $(409 \mathrm{~K})$ and high ionizing radiation environment testing also revealed successful and reliable operation of these MEMS devices over CMOS equivalents. Finally, microfabricated circuits such as multiplexers, 1-bit full adder and a 2-bit full adder that employ the $\mathrm{XOR}$ and AND gates was also presented here.

\section{ACKNOWLEDGEMENT}

This work is supported by DARPA NEMS grant \#NBCH1090003.

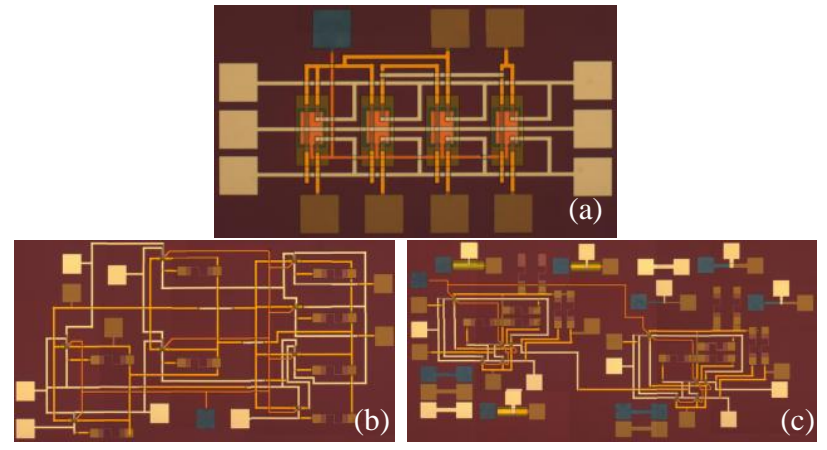

Figure 12: Single-device MEMS logic gates used to fabricate a)1-bit multiplexor using 4 AND gates b)1-bit adder using 3 $X O R \& 2 A N D$ gates and c)2-bit full adder made using 6 XOR \& 4 AND gates.

\section{REFERENCES}

[1] F. K. Chowdhury, K. N. Chappanda, D. Saab, and M. TabibAzar, "Novel Single-Device "XOR" and "AND" Gates for High Speed, Very Low Power LSI Mechanical Processors " in The 16th International Conference on Solid-State Sensors, Actuators and Microsystems Beijing, China, 2011.

[2] M. Tabib-Azar, S. R. Venumbaka, K. Alzoubi, and D. Saab, "1 volt, $1 \mathrm{GHz}$ nems switches," in Sensors, 2010 IEEE, 2010, pp. 1424-1426.

[3] K. Alzoubi, D. G. Saab, and M. Tabib-Azar, "Circuit simulation for Nano-Electro-Mechanical switches VLSI circuits," in Circuits and Systems (MWSCAS), 2010 53rd IEEE International Midwest Symposium on, 2010, pp. 1177-1180.

[4] V. K. Sirigir, K. Alzoubi, D. G. Saab, F. Kocan, and M. TabibAzar, "Ultra-low-Power Ultra-fast Hybrid CNEMS-CMOS FPGA," in Field Programmable Logic and Applications (FPL), 2010 International Conference on, 2010, pp. 368-373.

[5] M. Tabib-Azar, K. Alzoubi, and D. Saab, "Novel MEMS 900 $\mathrm{MHz}$ electrostatic silicon delay line," in Sensors, 2010 IEEE, 2010, pp. 205-207.

[6] K. Akarvardar, D. Elata, R. Parsa, G. C. Wan, K. Yoo, J. Provine, P. Peumans, R. T. Howe, and H. S. P. Wong, "Design Considerations for Complementary Nanoelectromechanical Logic Gates," in Electron Devices Meeting, 2007. IEDM 2007. IEEE International, 2007, pp. 299-302.

[7] R. Parsa, K. Akarvardar, J Provine, D. Lee, D. Elata, S. Mitra, H. S. P. Wong, and R. T. Howe, "Composite polysilicon-platinum lateral nanoelectromechanical relays " in 14th Solid-State Sensors, Actuators, and Microsystems Workshop Hilton Head, South Caroline, 2010, , pp. pp. 7 - 10

[8] L. Tsu-Jae King, J. Jaeseok, R. Nathanael, K. Hei, V. Pott, and E. Alon, "Prospects for MEM logic switch technology," in Electron Devices Meeting (IEDM), 2010 IEEE International, 2010, pp. 18.3.1-18.3.4.

[9] N. Sinha, T. S. Jones, G. Zhijun, and G. Piazza, "Body-biased complementary logic implemented using AIN piezoelectric MEMS switches," in Electron Devices Meeting (IEDM), 2009 IEEE International, 2009, pp. 1-4.

[10] G. M. Rebeiz, RF MEMS: theory, design, and technology: LibreDigital, 2003.

\section{CONTACT}

*M. Tabib-Azar, azar.m@utah.edu 